\title{
Preparation and Properties of PHEA/Chitosan Composite Hydrogel
}

\author{
Jeong Hoon Kim, Sang Jun Sim, Dong Hyun LeE, Dukjoon Kim, Young Kwan LeE, \\ Dong June CHUNG, and Ji-Heung $\mathrm{KIM}^{\dagger}$
}

Division of Applied Chemistry and Chemical Engineering, Polymer Technology Institute, Sungkyunkwan University, 300 Chunchun, Suwon, Kyunggi 440-746, Korea

(Received June 10, 2004; Accepted September 9, 2004; Published December 15, 2004)

\begin{abstract}
Biodegradable hydrogels based on synthetic PHEA and IPN type composite hydrogel with chitosan, a natural carbohydrate polymer, were prepared by crosslinking using glutaraldehyde in aqueous mixture. Relatively strong hydrogels were formed in a shaped mold. Preparation of crosslinked gels and their swelling behavior depending on the composition, medium, and $\mathrm{pH}$ of solution are investigated. The porous morphology of scaffolds, obtained by freeze-drying method, was observed by scanning electron microscopy. Also preliminary results on hydrolytic degradation and cytotoxicity test of the hydrogel are included. [DOI 10.1295/polymj.36.943]

KEY WORDS Poly(hydroxyethyl aspartamide) / Hydrogel / Chitosan / Biodegradation / Swelling Behavior / Scaffold / pH-Sensitive /
\end{abstract}

The importance of biodegradable polymers and hydrogel materials is being increasingly recognized and extensive studies have been conducted on their uses in various biomedical applications. ${ }^{1,2}$ Hydrogels based on both natural and synthetic polymers have continued to be of interest for encapsulation of drugs and cells, and most recently such hydrogels have become especially attractive to the new field of 'tissue engineering' as matrices for repairing and regenerating a wide variety of tissues and organs. ${ }^{3-5}$

Poly(amino acid)s, which have protein-like linkages, are known to be biodegradable and have been investigated in various biomedical applications including drug delivery systems. Poly(aspartic acid), PASP, is one of synthetic water-soluble and biodegradable polymer, which can be produced from the hydrolysis of polysuccinimide (PSI), ${ }^{6-8}$ the thermal condensation polymer of aspartic acid monomer. PASP has been commercialized as a biodegradable polymeric dispersant, and also investigated as a biodegradable superabsorbent in the crosslinked form or as a polymeric drug carrier. ${ }^{9,10} \alpha, \beta$-Poly $(N-2$-hydroxyethyl-DL-aspartamide), PHEA, is another important polymer, derived by coupling PSI with ethanolamine, which has been proposed as a potential plasma extender and carrier for macromolecular prodrugs. ${ }^{8,11,12}$ The presence of hydroxyl groups in the side chain provides the possibility of forming a covalent linkage between the polymer and drug molecules with or without proper spacer group. This polymer has been demonstrated to possess suitable physico-chemical characteristics for development of macromolecular prodrugs, including biodegradability, high water solubility, excellent biocompatibility, and low cost. Cross- linking of PHEA to prepare the corresponding gel can be accomplished by means of both chemical and physical processes. Recently, Giammona et al. has reported on the properties of hydrogels, which were obtained by gamma-irradiation of PHEA or modifiedPHEA with methacryloyl groups. ${ }^{13,14}$ Also we have reported on the preparation of PHEA based chemical hydrogels by two different methods and discussed their gel properties. ${ }^{15,16}$ As shown in our previous work, PHEA hydrogel could be simply made in aqueous system and exhibited some of interesting and desirable properties for biomedical application, but the hydrogel showed relatively weak mechanical strength and the in-situ formation of gel in a mold was hardly achieved.

Chitosan derived by $\mathrm{N}$-deacetylation of chitin is a biodegradable and biocompatible polymer. It has been shown recently that partially deacetylated chitin can be hydrolyzed by enzymes. Because of its biocompatibility, antibacterial properties, and remarkable affinity to proteins, it has been found to increase applications in areas such as hematology, immunology, wound healing, drug delivery, and cosmetics. In particular, The amino group, which is rare in polysaccharides, of chitosan influences the $\mathrm{pH}$-responsive behavior because $\mathrm{pH}$-sensitive hydrogels usually contain either acid or basic pendent groups in the network. ${ }^{17-21}$

In the present work, it was interesting to combine a natural $\mathrm{pH}$-sensitive chitosan and a synthetic PHEA to modify the gel properties of the component polymers. Biodegradable homo and composite hydrogels based on PHEA and chitosan were prepared chemically in aqueous medium. The characterization of these IPN

${ }^{\dagger}$ To whom correspondence should be addressed (E-mail: kimjh@skku.edu). 


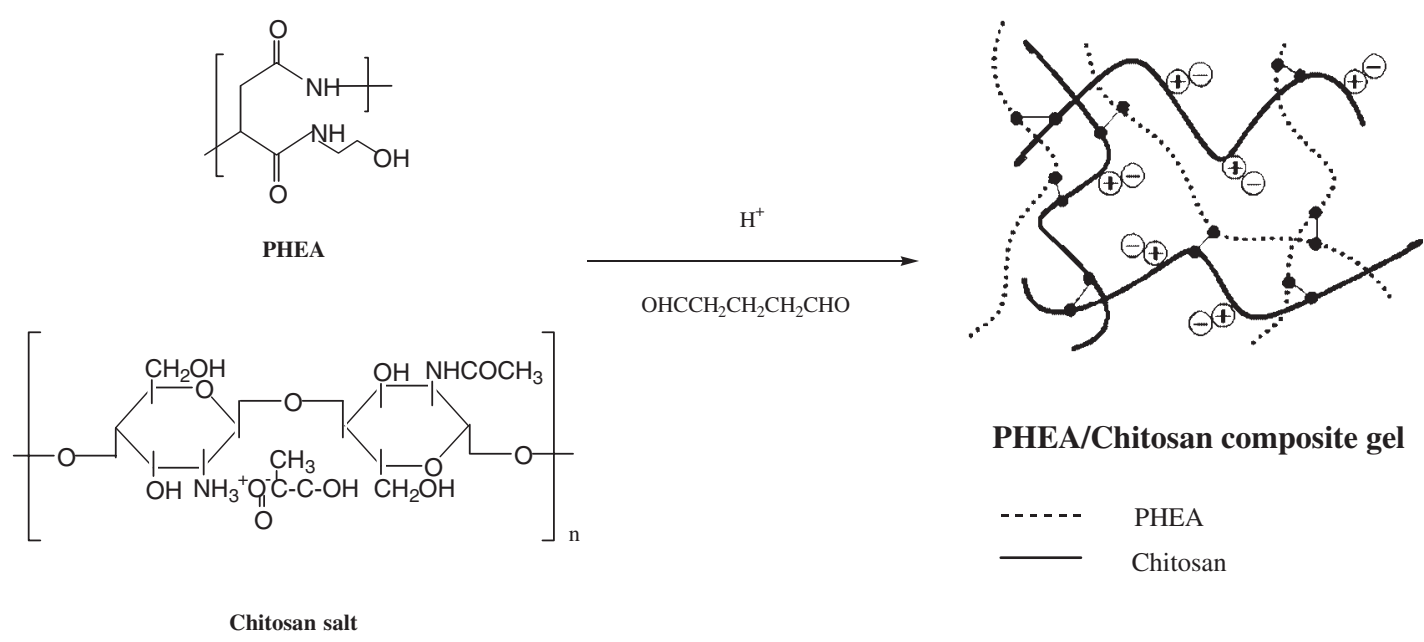

Scheme 1. Reaction scheme for the preparation of composite hydrogel.

type composite gels and their swelling properties are discussed.

\section{EXPERIMENTAL}

\section{Chemicals and Measurements}

L-Aspartic acid (98+\%), o-phosphoric acid $(98+\%)$, and ethanolamine $(99 \%)$ were purchased from Aldrich Chemical Co. and used as received. $N, N$-Dimethylformamide (DMF) was dried over $\mathrm{CaH}_{2}$ and fractionally distilled under reduced pressure. Chitosan sample $\left(M_{\mathrm{w}}=1.2 \times 10^{5}\right.$, deacetylation degree $>80 \%$ ) in its lactic acid salt form was provided by EZ Life Science Co. (Korea). All the other chemicals purchased were reagent grade and used without purification.

IR spectra were obtained on a PerkinElmer FT IR spectrometer (Model SPECTRUM 2000). ${ }^{1} \mathrm{H}$ NMR spectra were taken on a Varian Unity Inova 500 $\mathrm{MHz}$ Spectrometer. The thermal analysis was carried out on a PerkinElmer DSC/TGA7 Series thermal analysis system. Morphology of the prepared scaffolds was observed by SEM (FESEM Model JSM6700F, JEOL Inc.) to investigate the pore structure and pore size. Porous gel sample were mounted on the metal stub with double-sided tape and coated with $\mathrm{Au}-\mathrm{Pd}$ for $60 \mathrm{~s}$ under argon atmosphere using plasma sputter (Sputter Coater 108auto, Cressington Scientific Instruments Inc.).

\section{Preparation of Crosslinked Gels}

Hydrogels based on PHEA and commercial chitosan were prepared by crosslinking reaction in aqueous system using glutaraldehyde as the crosslinking reagent. 10 or $20 \mathrm{wt} \%$ aqueous solution with different composition was prepared and degassed by repeated vacuum and nitrogen purges. Into the polymer solu- tion was added glutalaldehyde $(10 \mathrm{~mol} \%)$ and catalytic $\mathrm{HCl}$, and the reaction mixture was placed at $40^{\circ} \mathrm{C}$ for $5 \mathrm{~h}$. The gel sheets could be formed in a glass mold and washed in excess water before being freeze-dried in vacuum.

\section{Measurement of Swelling Degree}

The swelling degree and rate in different media were measured by using a simple tea-bag method or by direct weighing of the swollen gel. A certain amount of dry gel disc or sheet $\left(W_{\text {dry }}\right)$ was weighed (in an empty bag), and then placed in an aqueous medium until near equilibrium swelling was obtained. The weight of the swollen gel ( $\left.W_{\text {swell }}\right)$ was measured directly or was obtained by subtracting the predetermined empty wet tea-bag weight from the total weight of bag including gel. The swelling ratio was simply calculated using the equation below;

$$
\text { Swelling Ratio }=W_{\text {swell }} / W_{\text {dry }} \times 100
$$

\section{Cytotoxicity Test}

To evaluate cytotoxicity and biocompatibility of the prepared hydrogels, L929 fibroblasts cell lines were used. The hydrogels were cut into $\pi \times 1.3 \times 1.3 \mathrm{~cm}^{2}$ pieces and put in 6-well plates with tissue culture polystyrene (TCPS) as a control substrate. All hydrogels were sterilized with $70 \%$ ethyl alcohol and UV irradiation, and then the samples were washed with phosphate buffered saline (PBS). L929 fibroblasts cells were cultured in the growth medium which was made up of Dulbecco's modified Eagle's medium (DMEM) supplemented with $10 \%$ (v/v) fetal bovine serum (FBS) at $37^{\circ} \mathrm{C}$ in a $5 \% \mathrm{CO}_{2}$ atmosphere for 3 d. L929 fibroblasts were purchase from Korea cell line bank. The cell response was observed everyday with a microscope. 


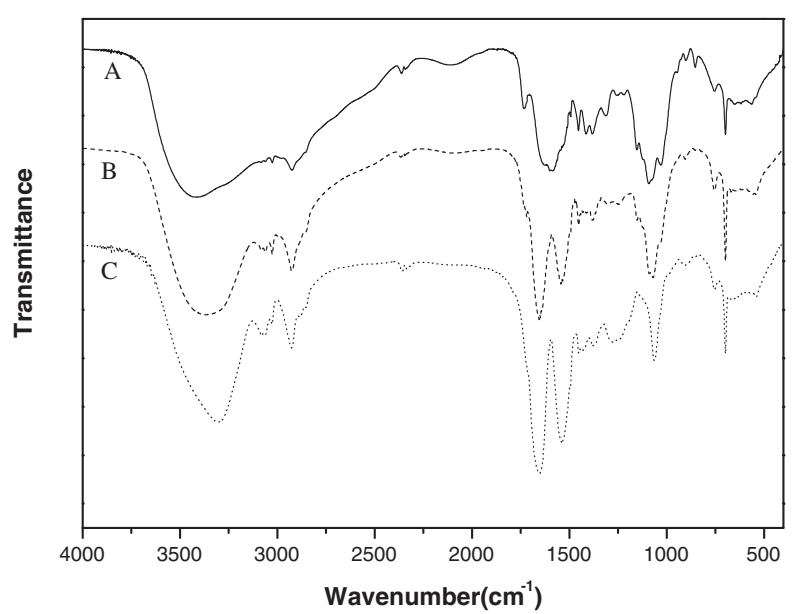

Figure 1. FT IR spectra of (A) Chitosan, (B) Composite gel, (C) PHEA.

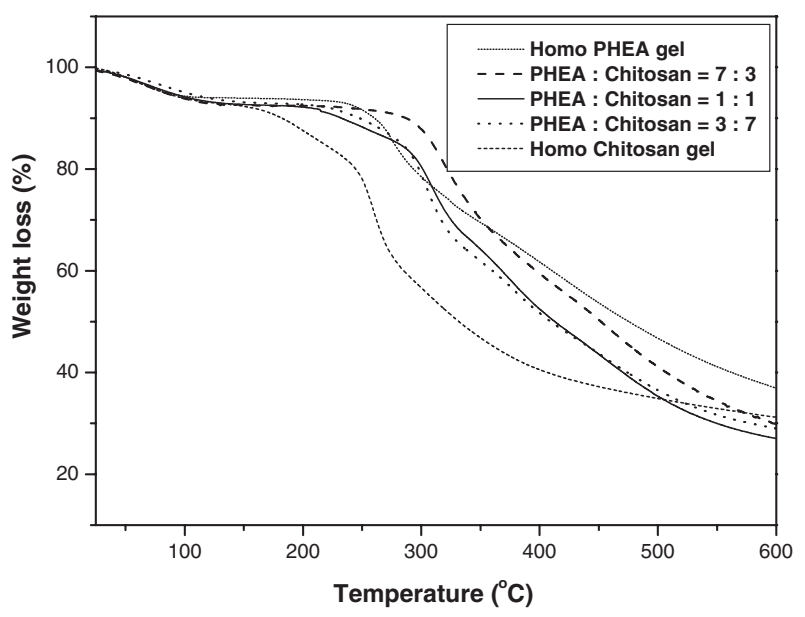

Figure 2. TGA thermograms of crosslinked gels.

\section{RESULTS AND DISCUSSION}

\section{Preparation and Characterization of Crosslinked Gels}

The preparation of polysuccinimide, the precursor polymer, has already been described in previous literature. ${ }^{8,14}$ The polymer possessed a reduced viscosity of $38 \mathrm{~mL} / \mathrm{g}$ in DMF. The molecular weight was estimated to be about $120,000 \mathrm{Da}$, as calculated from an empirical equation relating the solution viscosity to the molecular weight. ${ }^{5}$ PHEA was prepared from PSI via reaction with ethanolamine using a literature method known. The prepared PHEA was purified by dialysis and freeze-dried to obtain a white powder. Homo PHEA hydrogel or composite hydrogel with chitosan was prepared by chemical crosslinking in aqueous system using glutaraldehyde. The presence of hydroxyl groups on both polymers and additional amine groups on the chitosan should participate in the crosslinking reaction to provide IPN type compo-

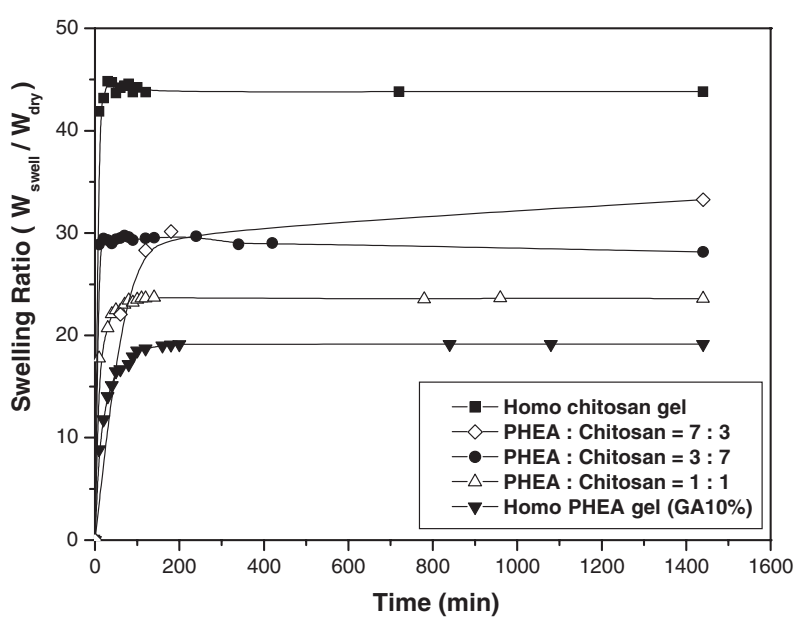

Figure 3. Typical swelling curves of gels in pure water at $25^{\circ} \mathrm{C}$.

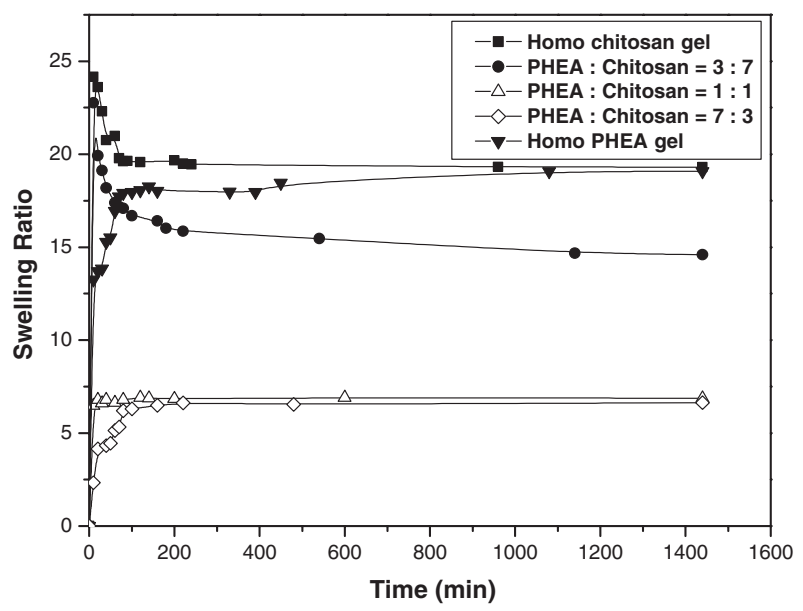

Figure 4. Typical swelling curves of gels in PBS (pH 7.4) at $25^{\circ} \mathrm{C}$.

site hydrogel material. For the preparation of porous scaffold, the swelled gel was frozen in liquid nitrogen and liphophilized by freeze-drying.

Figure 1 shows IR spectra of both homo PHEA and chitosan gel, and 1:1 composite gel, respectively. The spectrum A shows characteristic bands of saccharide structure at 897 and $1153 \mathrm{~cm}^{-1}$, and a strong amino characteristic peak at around $1591 \mathrm{~cm}^{-1}$, plus additional weak absorption bands at $1735 \mathrm{~cm}^{-1}$ corresponding to lactate moiety. The spectrum C of PHEA hydrogel shows very strong and characteristic amide bands at 1610 and $1530 \mathrm{~cm}^{-1}$ with broad hydroxyl absorption band at $3200-3600 \mathrm{~cm}^{-1}$. The mid spectrum B of composite gel possesses combined absorption bands of both polymer components. From the TGA thermograms of the prepared gels (Figure 2), homo PHEA and the composite hydrogels were found to be stable up to about $220^{\circ} \mathrm{C}$ in nitrogen and their thermal stability was higher than chitosan itself. 


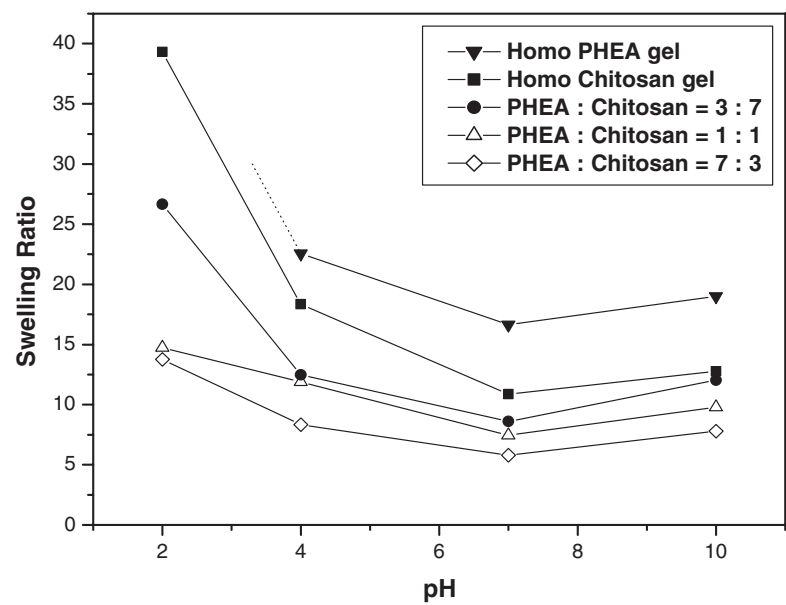

Figure 5. Swelling dependence on the $\mathrm{pH}$ of buffer solutions at RT (Sample: composite gel [from 10\% solution]).

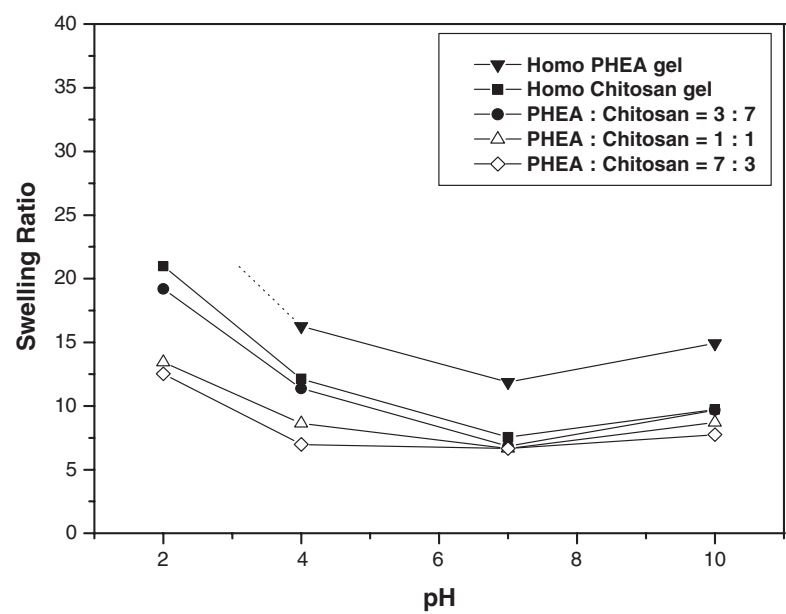

Figure 6. Swelling dependence on the $\mathrm{pH}$ of buffer solutions at RT (Sample: composite gel [from $20 \%$ solution]).

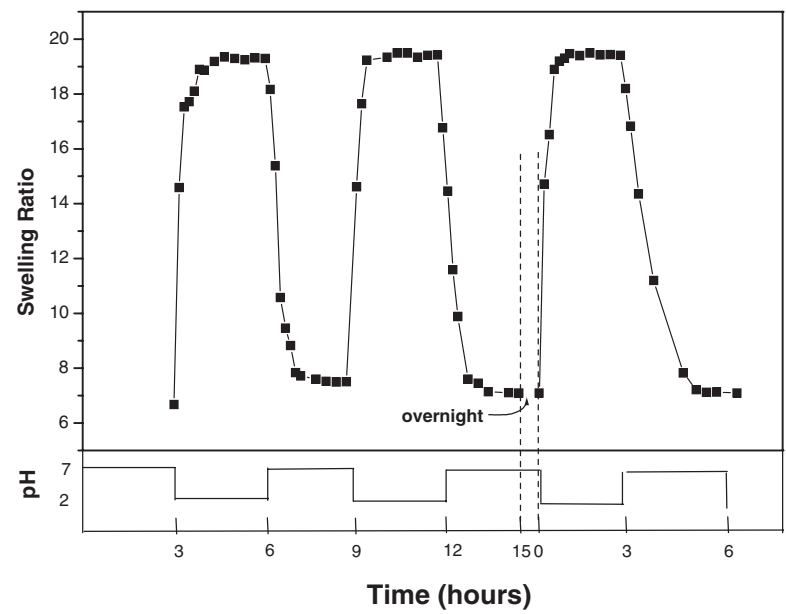

Figure 7. Swelling ratio of hydrogel as a function of time under repeated abrupt change of $\mathrm{pH}$ between $\mathrm{pH} 7$ and $\mathrm{pH} 2$ (Sample: composite gel (PC37), cast from 20\% solution).

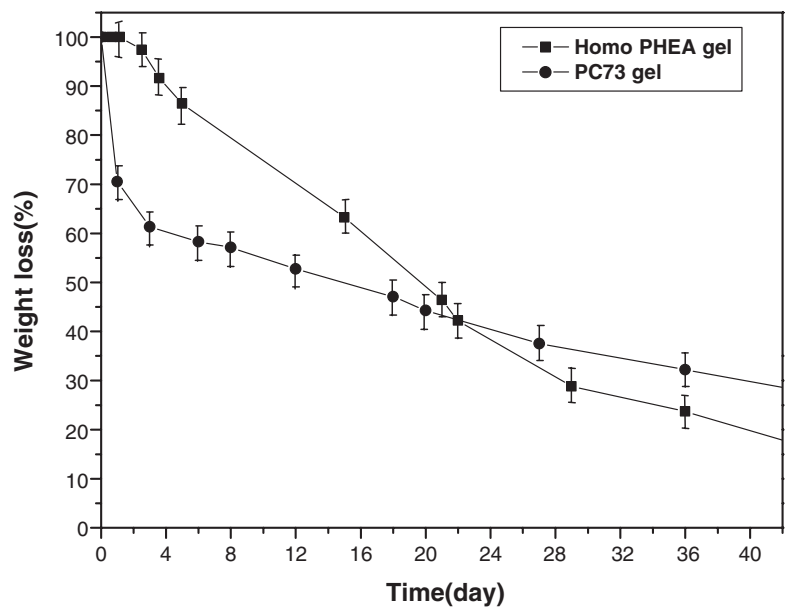

Figure 8. Hydrolytic degradation in PBS (pH 7.4) at $37^{\circ} \mathrm{C}$ (Sample: homo PHEA gel and PC73 composite gel).

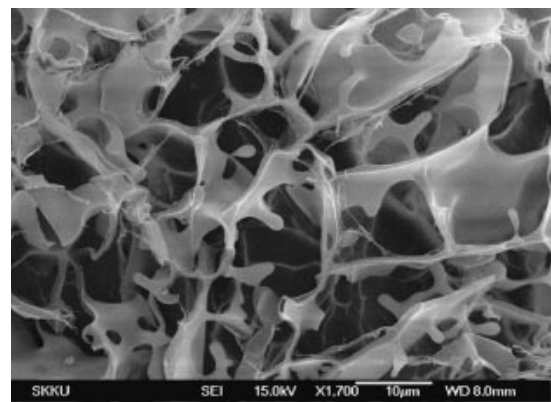

(a) Surface

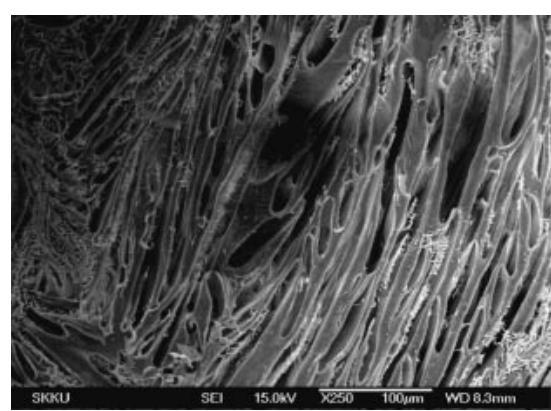

(b) Cross section

Figure 9. SEM images of freeze-dried composite hydrogel (PC73 composite gel).

\section{Swelling Behavior of PHEA/Chitosan Gels}

The prepared gels were tested as regards their swelling in aqueous solutions. Figure 3 shows water swelling curve for the different gel samples. The initial fast swelling seemed to reach equilibrium in $2-3 \mathrm{~h}$ and the swelling degree remained almost constant thereafter. The swelling degrees were measured to be in the range 20-40 g water/g dry gel, where PHEA gel was in the lowest, the chitosan in the highest, and the composite gels were in between. The swelling degree was increased as the content of more hydrophilic chitosan in- 
(a)
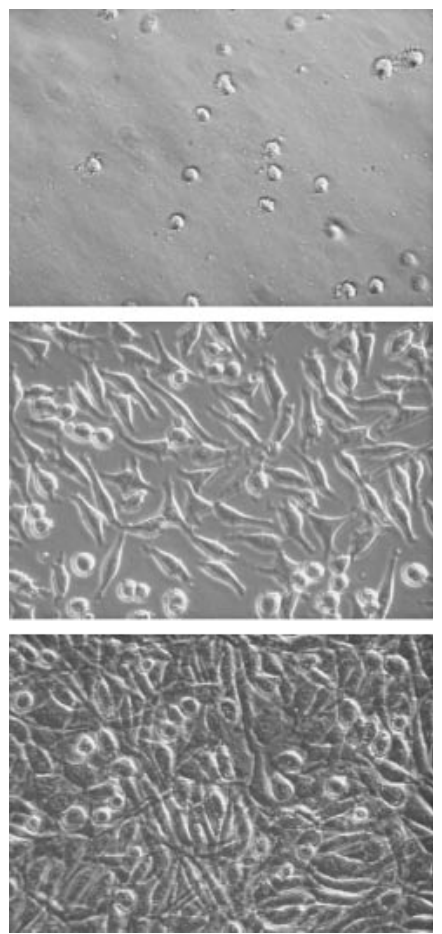

(b)
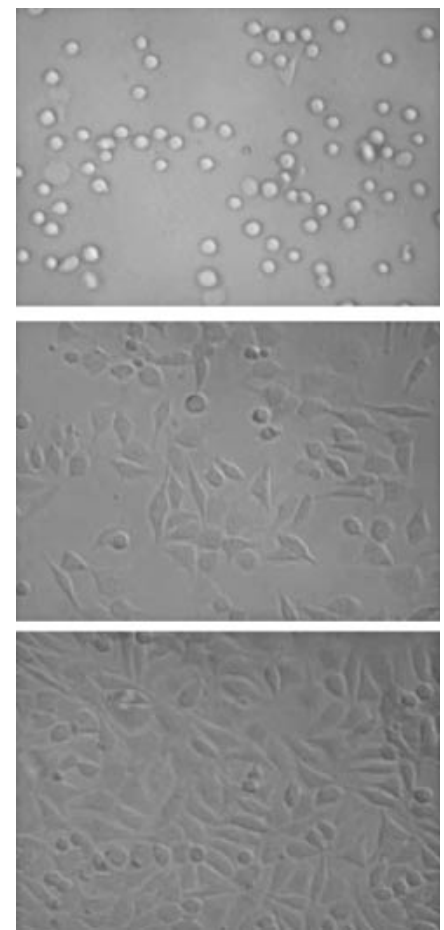

(c)
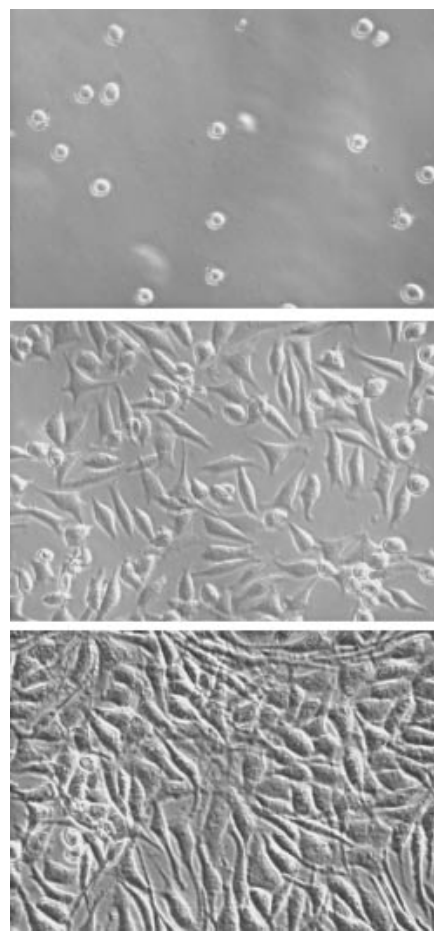

$12 \mathrm{~h}$

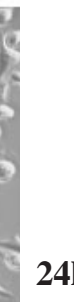

Time

$24 h$

$48 \mathrm{~h}$

Figure 10. Microscopy photographs of the L929 cells 12, 24 and $48 \mathrm{~h}$ after they were plated on: (a) TCPS (b) Homo PHEA gel (c) Composite gel (PC11) (Culture condition: $37.0{ }^{\circ} \mathrm{C}, 5.0 \% \mathrm{CO}_{2}, 3 \mathrm{~mL}$ DMEM with FBS (10\%)).

creased. Figure 4 shows swelling degrees in PBS solutions. The overall swelling degrees were lower compared to those in pure water, probably caused by the ionic nature of chitosan component. The swelling degree of nonionic PHEA gel, however, remained almost at the same level. On the other hand, the swelling speed was observed to be faster as the chitosan content was increased. So-called "overshooting" phenomena was observed in homo chitosan and chitosan-rich composite gel (PC37) as can be found in some hydrogel systems. The reason of this behavior might be attributed to the kinetic effects between molecular relaxation of the network and fast diffusion into the gel before the swelling reach the equilibrium value. ${ }^{22}$

Figure 5 and 6 show pH-sensitive characteristics of hydrogel, which are investigated by swelling test under various $\mathrm{pH}$ values ranging between 2 and 10 . The data shown in Figures 5 and 6 were obtained from gel samples prepared in $10 \mathrm{wt} \%$ and $20 \mathrm{wt} \%$ aqueous solution, respectively. As can be seen, overall swelling degrees are relatively low in samples from thick $20 \mathrm{wt} \%$ solution than those from $10 \mathrm{wt} \%$ solution, due to the difference in crosslinking density. Throughout the entire compositions, however, the swelling degrees were higher at lower $\mathrm{pH}$, i.e., in acidic medium. As the $\mathrm{pH}$ value increases, the swelling degree tended to decrease upto neutral $\mathrm{pH} 7$, and then the swelling degrees slightly increased at alkaline $\mathrm{pH}$.
In particular, the homo PHEA hydrogel was found to be unstable at low $\mathrm{pH}$ of 2 , suggesting gel decomposition at this strongly acidic condition. As is well known, the $\mathrm{pH}$ sensitivity will mainly affected by chitosan amino groups. ${ }^{15-18}$ Figure 7 shows the pulsatile swelling behavior of one exemplary composite hydrogel (PC37, PHEA:Chitosan $=3: 7$ by weight) at $25^{\circ} \mathrm{C}$ with solution $\mathrm{pH}$ values alternating between 2 and 7 . A rapid swelling and deswelling behavior was observed and the process was proved to be repeatable.

\section{Hydrolytic Degradation of PHEA and Composite Gel}

Aspect of gel degradation in an aqueous environment was investigated by monitoring the weight loss of the material as a function of immersion time. Typical results are plotted in Figure 8. A series of swollen gels within tea-bags was placed in a buffered solution of $\mathrm{pH} 7.4$ controlled at $37^{\circ} \mathrm{C}$, and sampled out after a given period of time to check the water absorbency and weight loss. The weight was measured by freeze-drying of the remaining gel. Under hydrolytic condition, no appreciable change in weight occurred during the initial 2-3d for homo PHEA gel, but a gradual decrease, at an almost constant rate over a relatively long period (about 2 months), was observed thereafter. In comparison, the composite gel (PC73) showed initial drop in weight within about 3-4d, which might be ascribed to the dissolution of soluble 
fraction trapped within the gel along with lactate moiety. After that, the gel degradation seemed to proceed gradually as time over a long period of time. The homo chitosan gel also exhibited similar weight loss pattern as above composite gel. The shape of the swollen gel, however, remained almost unchanged during the measuring period.

\section{Morphology of Gel Scaffold and Cytotoxicity Test}

Recently, hydrogels have become increasingly studied as matrices for tissue engineering. ${ }^{3-5}$ PHEA-based hydrogel also has a potential for this purpose because of the several advantageous properties. This hydrogel possesses biodegradability and potential biocompatibility, chemical modification is viable using hydroxyl pendant, and relatively strong gel with different porosity might be possible by changing crosslinking density using proper crosslinking agent. PHEA compoite hydrogels prepared in this work could be shaped into porous scaffold by simple freeze-drying methods of water-swollen gel. Figure 9a shows representative SEM micrographs of gel sample (PC73 gel in sheet form) indicating microporous structure of the scaffold possessing an interconnected network structure composed of a thin wall with several tens and hundreds micron pores. Figure $9 \mathrm{~b}$ shows the cross-section of gel sheet with elongated pore textures.

To evaluate the cytotoxicity and biocompatibility of prepared hydrogels, L929 fibroblasts cell line was used. The cellular behavior on a biomaterial is an important factor determining its biocompatibility. L929 fibroblast cell line was cultured for $3 \mathrm{~d}$ and observed the surface morphology by optical microscope (Figure 10). The surfaces of homo and composite hydrogel were shown with similar cell growth pattern compared to the surface of TCPS control and improved cell growth on the hydrogel surface because of enhanced hydrophilicity compared to the control surface was observed. Thereby, PHEA homo and composite hydrogels were considered to be a quite biocompatible material.

Acknowledgment. This work was supported by the Korea Research Foundation Grant (KRF-2004-005D00070).

\section{REFERENCES}

1. A. J. Domb, J. Kost, and D. M. Wiseman, Ed., "Handbook of Biodegradable Polymers," Harwood Academic Publishers, Amsterdam, 1997.

2. S. Dumitriu, Ed., "Polymeric Biomaterials, 2nd ed.," Marcel Dekker, New York, N.Y., 2002.

3. H.-W. Kang, Y. Tabata, and Y. Ikada, Biomaterials, 20, 1339 (1999).

4. K. Y. Lee and D. J. Mooney, Chem. Rev., 101, 1869 (2001).

5. A. S. Hoffman, Adv. Drug Delivery Rev., 43, 3 (2002).

6. S. K. Wolk, G. Swift, Y. H. Paik, K. M. Yocom, R. L. Smith, and E. S. Simon, Macromolecules, 27, 7613 (1994).

7. T. Nakato, M. Yoshitake, K. Matsubara, M. Tomida, and T. Kakuchi, Macromolecules, 31, 2107 (1998).

8. P. Neri, G. Antoni, F. Benvenuti, F. Colola, and G. Gazzei, J. Med. Chem., 16, 893 (1972).

9. J. D. Andrade, "Hydrogels for Medical and Related Application," ACS Symp. Ser. No. 631, American Chemical Society, Washington, D.C., 1996.

10. J.-H. Kim, J. H. Lee, and S.-W. Yoon, J. Ind. Eng. Chem., 8, 138 (2002).

11. G. Pitarresi, V. Tomarchio, G. Cavallaro, and G. Giammona, J. Bioact. Compat. Polym., 11, 328 (1996).

12. G. Caldwell, E. W. Nense, and A. G. Perlwitz, J. Appl. Polym. Sci., 66, 911 (1997).

13. G. Pitarresi, M. Licciardi, G. Cavallaro, G. Spadaro, and G. Giammona, Colloid Polym. Sci., 279, 579 (2001).

14. G. Giammona, G. Pitarresi, V. Tomarchio, and G. Spadro, Colloid Polym. Sci., 272, 12 (1994).

15. S.-W. Yoon, D. J. Chung, and J.-H. Kim, J. Appl. Polym. Sci., 90, 3741 (2003).

16. J. H. Kim, S. J. Sim, D. H. Lee, D. J. Kim, Y. K. Lee, and J.-H. Kim, J. Ind. Eng. Chem., 10, 278 (2004).

17. S. J. Kim, S. J. Park, and S. I. Kim, React. Funct. Polym., 55, 53 (2003).

18. F. Yao, W. Chen, C. Liu, and K. D. Yao, J. Appl. Polym. Sci., 89, 3850 (2003).

19. X. Qu, A. Wirsen, and A.-C. Albertsson, J. Appl. Polym. Sci., 74, 3186 (1999).

20. X. Qu, A. Wirsen, and A.-C. Albertsson, Polymer, 41, 4589 (2000).

21. C. H. Kim and K. S. Choi, J. Ind. Eng. Chem., 8, 71 (2002).

22. W.-F. Lee and C.-F. Chen, J. Appl. Polym. Sci., 69, 2021 (1998). 